\title{
THE PROPAGATION OF FAST MAGNETOACOUSTIC WAVES NEAR A ROTATING BLACK HOLE
}

\author{
JUNYA ABE AND MASAYOSHI YOKOSAWA \\ Ibaraki University \\ Bunkyou Mito, Japan
}

We belive that Active Galactic Nucleis(AGNs) have one or a few black holes in the center and get the activity from the binding energy of the matter falling into the black hole or(and) the rotational energy of the black hole. Since the sources of their energy exist near the black hole, the energy have to be carried from the vincity of black hole to a distance by some ways. As one of the way, we study the propagation of the waves (ex. The case of the light waves, Hanni 1977, and of sound wave, Takahashi et al. 1990). We investigated the propagation of fast magnetoacoustic wave. We belive the collimation of jet are caused by magnetic field. Further more, we think that the waves can extract the rotational energy of the black hole. This process is the version of wave of Penrose process(Penrose 1968), and is called the super radiant scattering.

In magnetic fluid, the equations of the propagation of the magnetoacoustic waves were given by Licherowicz(1967, the equation (35-27)). Here we use the units of $c=G=1$. We divided the equation of Licherowicz into two equations of fast and slow magnetoacoustic waves,

$$
\begin{aligned}
& f^{\alpha \beta} \psi_{, \alpha} \psi_{, \beta}=0, \\
& s^{\alpha \beta} \psi_{, \alpha} \psi_{, \beta}=0,
\end{aligned}
$$

where $\psi$ is called eikonal and the each surface of $\psi=$ constant expresses a hypersurface of space-time. $f^{\alpha \beta}$ and $s^{\alpha \beta}$ are given by the quantities of fluid, magnetic field and gravitational field. When the sound velocity in the fluid is much smaller than the light velocity, $f^{\alpha \beta}$ and $s^{\alpha \beta}$ are approximately given by the following equations,

$$
\begin{aligned}
f^{\alpha \beta} & \approx g^{\alpha \beta}+\frac{n h}{B^{2}} u^{\alpha} u^{\beta}, \\
s^{\alpha \beta} & \approx 0 .
\end{aligned}
$$


where $g^{\alpha \beta}$ is the metric of space-time, $n$ is the rest mass energy density of the fluid, $h$ is the specific enthalpy of the fluid, $B^{2}$ is the square of the intesity of magnetic field and $u^{\alpha}$ is the four velocity of the fluid.

We investigated the prpoagation of the fast magnetoacoustic waves near a black hole. Therefore the metric of space-time is given by the black hole(Kerr metric; Boyer-lindquist coordinate). In the case that the fluid uniformly radially falls in a black hole and the magnetic field is uniform (Wald 1974), we seach the propagation of waves.

Figure 1 show the propagation of wave front of the fast magnetoacoustic wave on the equator surface $(\theta=\pi / 2)$. The black hole is the extrem Kerr black hole (the event horizon is a sphere with a radius of $1 m$; here $m$ is the mass of black hole), and the source of the wave is located at $r=2 m, \phi=0$.

Seeing Figure 1, we understand the wave front can't propagate to the event horizon. This reason is that the propagation in the radial direction depends on the value of $f^{r r}$. When $f^{r r}=0$, the velocity in the radial direction is zero. Consequently, the waves radiated in the region of $f^{r r}<0$ can't propagate in the region of $f^{r r}>0$ and the oposite is also true. In this case, on the position, $r \approx 1.6 m, f^{r r}=0$. So the waves radiated far away from black hole can't approach to the black hole and the waves radiated near the black hole can't propagate in the outer distance. This shows that in this case, the fast magnetoacoustic waves can't propagte the energy existing the vicinity of black hole to the outer distance and can't extract much rotaional energy of black hole. In the future we'll investigate the propagation of the fast magnetoacoustic waves in the another case.

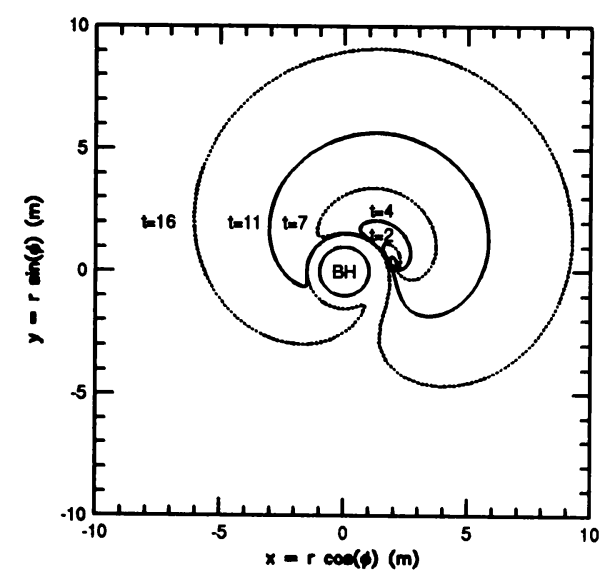

Figure 1. The wave front of fast magnetoacoustic wave; The source of wave is located at $r=2 m, \phi=0, \theta=0(x=2 m, y=0$, $z=0)$. The center circle with radius of $1 \mathrm{~m}$, shows the event horizonof the black hole. Here $m$ is the mass of the balck hole. The other lines show the wave fronts, and the each time of the wave fronts, is $t=1,2,4,7,11$, and 16 , respectively. Here the black hole is the extreme Kerr black hole and rotate in the positive $\phi$-direction. 